\title{
Using Ex-Situ TEM Studies to Gain Fundamental Insights into Bimetallic Nanoparticles
}

\section{Chris Kliewer}

ExxonMobil Research, Annandale, New Jersey, United States

Bimetallic nanoparticles are frequently used in catalytic reactions. In many cases, it is important that both metals remain accessible during the chemical reaction (i.e., some fraction of each metal must be present on the active surface). Hence it becomes critical to understand phenomena (process parameters) that may alter this critical morphology.In this study, model catalyst nanoparticles were created by depositing metal from a 50:50 $\mathrm{CuAu}$ nanowire onto a TEM grid. The high vacuum metal deposition was conducted using an Edwards E306A coater, and the support material was an A12O3-coated, 200 mesh, holey-carbon-coated mo TEM grid. Subsequent to deposition, the bimetallic particles were immediately transferred into a specially-designed, minaturized fixed bed reactor system (Figure 1),1-3 where the TEM grid was given an ex-situ reductive treatment $(500 \mathrm{C}, 99.999 \% \mathrm{H} 2,1 \mathrm{~h})$. The grid was subsequently transferred via an inert protocol 1-3 into a conventional TEM where it was examined in the bruight field (BF) imaging mode at an accelerating voltage of $200 \mathrm{kV}$. BF TEM images were collected from $\mathrm{CuAu}$ nanoparticles that were randomly-selected, and the imaged areas were "mapped" so that the identical structures could be reexamined subsequent to further ex-situ treatments. After the initial deposition and reduction, the $\mathrm{CuAu}$ nanoparticles appeared as small, "dark" features on the TEM grid (Figure 2a).Next, the TEM grid was then given an air oxidation $(200 \mathrm{C}$, laboratory air, 6h) and a final reduction $(300 \mathrm{C}, 99.999 \% \mathrm{H} 2,1 \mathrm{~h})$ treatment (Figure $2 \mathrm{~b}$ and Figure 2c, respectively). The grid was re-examined subsequent to the air oxidation. Significant morphological changes were clearly evident; the $\mathrm{CuAu}$ nanoparticles no longer appeared as the uniformly "dark" features observed after reduction but instead had transformed into coreshell structures having "light grey" exterior layers that surrounded "darker" cores, suggesting a manifestation of the Kirkendall effect. The core-shell features, however, were no longer apparent after the final reduction (Figure 2c).Elemental analyses from the "darker" particle cores suggested that these interior structures were preferentially enriched in $\mathrm{Au}$ (Figure 2d), while the "light grey" features were preferentially enriched in $\mathrm{Cu}$ (Figure 2e). Most importantly, a close examination of these features subsequent to the final reduction indicates the existence of a new population of "small", $\mathrm{Cu}$-rich metal particles (Figure 2f). 


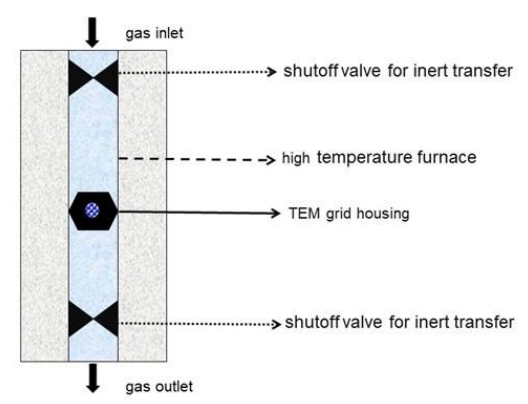

Figure 1. Figure 1: Schematic shows the basic design of the ex-situ reactor.

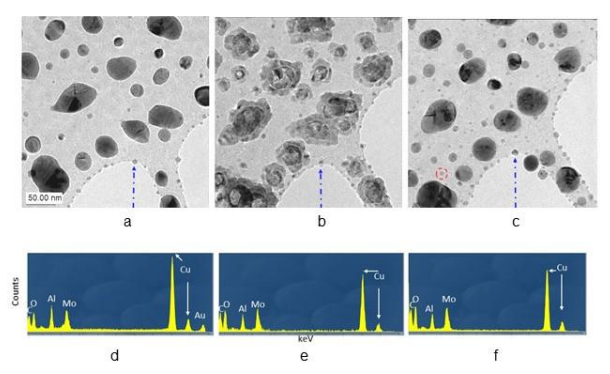

Figure 2. Figure 2: BF TEM images shows CuAu particles subsequent to: (a) 500C, 99.999\% H2, 1h, (b) 200C, lab air, 6h, and (c) 300C, 99.999\% H2, 1h treatments. EDS analyses indicate that the (d) "dark" interior core remains enriched in Au subsequent to the air oxidation treatment but that the (e) "light grey" exterior shell structure is preferentially enriched in $\mathrm{Cu}$. (f) A new population of small, $\mathrm{Cu}$-rich particles formed subsequent to the final reduction (red circle, area (c)).

References

1. C.E. Kliewer et.a. "Ex-Situ Transmission Electron Microscopy: A Fixed Bed Reactor Approach" Microscopy and Microanalysis (2006) 12 (2) 135

2. G. Kiss et. al. "Hydrothermal Deactivation of Silica-Supported Cobalt Catalysts in Fischer-Tropsch Synthesis" J. Catal. (2003) 217127.

3. C.E. Kliewer et. al. "Morphological Transformations During Fischer-Tropsch Synthesis on a TitaniaSupported Cobalt Catalyst" Catalysis Today (2019) 323233. 